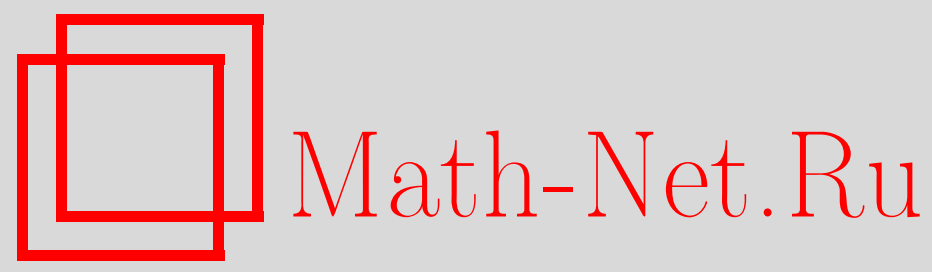

Г. С. Мустафаев, Банаховы алгебры с ограниченными группами образующих и свойство Шура, Матем. заметки, 2002, том 71, выпуск 5, 725-731

DOI: https://doi.org/10.4213/mzm380

Использование Общероссийского математического портала Math-Net.Ru подразумевает, что вы прочитали и согласны с пользовательским соглашением http://www.mathnet.ru/rus/agreement

Параметры загрузки:

IP: 52.6 .47 .48

26 апреля 2023 г., 14:20:13

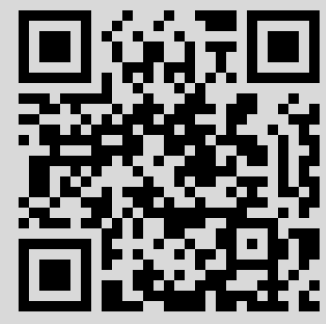




\section{БАНАХОВЫ АЛГЕБРЫ С ОГРАНИЧЕННЫМИ ГРУППАМИ ОБРАЗУЮШИХ И СВОЙСТВО ШУРА}

Г. С. Мустафаев

Напомним, что банахово пространство $X$ обладает свойством Шура, если всякое слабо компактное множество в $X$ является сильно компактным. В этой заметке рассматривается банахова алгебра $A$ с ограниченной группой образующих. Наряду с другими результатами устанавливается, что если $A^{*}$ обладает свойством Шура, то пространство Гельфанда алгебры $A$ есть вполне несовершенное множество и, кроме того, $A^{*}$ обладает свойством Радона-Никодима.

Библиография: 9 названий.

1. Введение и предварительные сведения. Хорошо известно, что в пространстве суммируемых последовательностей $l^{1}$ всякое слабо компактное множество является сильно компактньм. Банахово пространство $X$, обладающее этим свойством, назьвается пространством со свойством Шура. Пусть $(\Omega, \Sigma, \mu)$ - пространство с конечной мерой. Говорят [1, с. 61$]$, что $X$ обладает свойством Радона-Никодима, если для каждой $\mu$-непрерывной векторной меры $G: \Sigma \rightarrow X$ с ограниченной вариацией найдется $g \in L^{1}(\mu, X)$ такое, что

$$
G(E)=\int_{E} g d \mu \quad \text { для всех } E \in \Sigma .
$$

В дальнейшем нам понадобится следующий факт [1, с. 81-82]: если любое сепарабельное подпространство сопряженного пространства $X^{*}$ содержится в некотором сепарабельном сопряженном пространстве, то $X^{*}$ обладает свойством Радона-Никодима.

Пусть $G$ - локально компактная абелева группа, $K$ - компактное подмножество $G$ и $P M(K)$ - пространство псевдомер, сосредоточенных в $K . \Phi$. Лю-Пикар [2, теорема 1$]$ доказал, что если $K$ - вполне несовершенное множество, т.е. если оно не содержит непустого совершенного подмножества, то $P M(K)$ обладает свойством Шура (а также и свойством Радона-Никодима [3, теорема 2.8]). Если $K$ метризуемо, то верно и обратное утверждение [2, предложение 3]. В работе [4] дана некоторая характеризация пространств $P M(K)$ со свойством Шура. В частности, доказано, что если $P M(K)$ обладает свойством Шура, то $K$ - вполне несовершенное множество.

В этой заметке мы будем рассматривать коммутативные банаховы алгебры $A$ с ограниченными групшами образуюших. Наряду с другими результатами мы покажем, что если $A^{*}$ обладает свойством Шура, то пространство Гельфанда $\Sigma_{A}$ алгебры $A$ вполне несовершенное множество; кроме того, $A^{*}$ обладает свойством Радона-Никодима. 
Принятые здесь обозначения более или менее стандартны: $X$ - банахово пространство, $X^{*}$ - его сопряженное, $X^{* *}$ - его второе сопряженное и $X_{(1)}$ - замкнутьй единичный шар в $X$. Для $\varphi \in X^{*}$ и $x \in X$ через $\langle\varphi, x\rangle$ будем обозначать естественную двойственность между $X$ и $X^{*}$. Слабую топологию в $X$ и *-слабую топологию в $X^{*}$ будем обозначать через $w$ и $w^{*}$ соответственно. Через $\bar{E}$ будем обозначать замыкание по норме множества $E \subset X$.

Пусть $A$ - произвольная (комплексная) коммутативная банахова алгебра. Как известно [5], [6], в $A^{* *}$ можно определить умножение (после чего $A^{* *}$ становится банаховой алгеброй), которое является естественным продолжением исходного умножения в $A$. Это умножение определяется следующим образом: если $a, b \in A, \varphi \in A^{*}$ и $F, G \in A^{* *}$, то положим $\langle\varphi \cdot a, b\rangle=\langle\varphi, a b\rangle,\langle G \cdot \varphi, a\rangle=\langle G, \varphi \cdot a\rangle$ и $\langle F \cdot G, \varphi\rangle=\langle F, G \cdot \varphi\rangle$. Алгебра $A$ назьвается регулярной по Аренсу, если два естественных произведения, определенных на $A^{* *}$, совпадают. В дальнейшем алгебру $A$ будем считать подалгеброй $A^{* *}$. Напомним также, что $\varphi \in A^{*}$ назьвается слабо почти периодическим (почти периодическим) функиионалом, если $\left\{\varphi \cdot a \mid a \in A_{(1)}\right\}$ слабо предкомпактно (предкомпактно). Обозначим через $\operatorname{wap}(A)(\operatorname{ap}(A))$ множество всех слабо почти периодических (почти периодических) функционалов в $A$. Легко видеть, что $\operatorname{wap}(A)$ и $\operatorname{ap}(A)$ являются замкнутыми подпространствами в $A^{*}$. Как известно [6], регулярность по Аренсу алгебры $A$ равносильна выполнению равенства $\operatorname{wap}(A)=A^{*}$.

2. Свойства Шура и Радона-Никодима. Пусть $A$ - коммутативная банахова алгебра. Предположим, что в $A$ имеется группа $\Gamma$ обратимых элементов $A$ такая, что $\sup \{\|\gamma\|: \gamma \in \Gamma\}=C_{\Gamma}<\infty$ и $\overline{\operatorname{span}} \Gamma=A$. В этом случае будем говорить, что $A$ имеет ограниченную группу образующих. Пусть $G$ - локально компактная абелева группа, $A(G)$ - алгебра Фурье и $J_{K}$ - наименьший замкнутый идеал в $A(G)$ с компактной оболочкой $K$. Тогда фактор алгебра $A(G) / J_{K}$ имеет ограниченную группу образующих [4]. Заметим также, что $P M(K)$ является сопряженным к $A(G) / J_{K}$ пространстBOM.

Основным результатом этой заметки является следующая теорема.

Теорема 1. Пусть $A$ - коммутативная банахова алгебра с ограниченной группой образующих. Если $A^{*}$ обладает свойством Шура, то справедливы следующие утверәсдения:

а) $\Sigma_{A}$ - вполне несовершенное множсество;

b) $A^{*}=\overline{\operatorname{span}} \Sigma_{A}$;

c) $A^{*}$ обладает свойством Радона-Никодима.

Для доказательства этой теоремы нам понадобятся некоторые предварительные результаты.

Пусть $A$ - коммутативная банахова алгебра, $\Gamma$ - ограниченная группа образующих в $A$. Задавая в $\Gamma$ дискретную топологию, определим отображение $\omega: L^{1}(\Gamma) \rightarrow A$ равенством

$$
\omega(f)=\int_{\Gamma} f(\gamma) \gamma^{-1} d \gamma
$$

Нетрудно видеть, что $\omega$ есть непрерьвньй гомоморфизм с плотным образом. Отсюда легко следует, что $A$ регулярна (по Шилову). Если положить $\operatorname{sp}(\omega)=h(\operatorname{Ker} \omega)$, то легко видеть, что $\operatorname{sp}(\omega)$ есть замкнутое подмножество $\widehat{\Gamma}$-групшы характеров $\Gamma$. Стандартная 
техника банаховых алгебр $[7$, с. 80$]$ показьвает, что $\Sigma_{A}$ можно отождествить с $\operatorname{sp}(\omega)$. Если обозначить через $\hat{a}$ преобразование Гельфанда элемента $a \in A$, то легко видеть, что

$$
\widehat{\omega(f)}(\chi)=\widehat{f}(\chi), \quad \chi \in \operatorname{sp}(\omega),
$$

где $\hat{f}$ - преобразование Фурье $f \in L^{1}(\Gamma)$.

Для произвольного $\varphi \in A^{*}$ положим $\widetilde{\varphi}=\omega^{*} \varphi$. Очевидно, что $\widetilde{\varphi} \in L^{\infty}(\Gamma), \widetilde{\varphi}(\gamma)=$ $\langle\varphi, \gamma\rangle$ и $\|\widetilde{\varphi}\|_{\infty} \leqslant C_{\Gamma}\|\varphi\|$. Заметим, что $\widetilde{\varphi \cdot s}=\widetilde{\varphi}_{s}(s \in \Gamma)$, где $\widetilde{\varphi}_{s}$ есть сдвиг функции $\widetilde{\varphi}$.

А теперь рассмотрим следующее множество:

$$
S=\left\{\varphi \in A^{*} \mid \widetilde{\varphi} \in \operatorname{AP}(\Gamma)\right\}
$$

где $\mathrm{AP}(\Gamma)$ есть пространство почти периодических функций. Нетрудно видеть, что $S$ является замкнутьп подпространством в $A^{*}$. Заметим, что если $\varphi \in S$, то $\varphi \cdot a \in S$

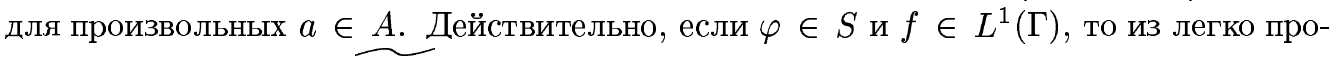
веряемого равенства $\widehat{\varphi \cdot \omega(f)}=\widetilde{\varphi} * f$ следует, что $\varphi \cdot \omega(f) \in S$. А в силу плотности $\left\{\omega(f), f \in L^{1}(\Gamma)\right\}$ в $A$ получим, что $\varphi \cdot a \in S$ для произвольных $a \in A$.

Лемма 1. Имеют место включения $\overline{\operatorname{span}} \Sigma_{A} \subset \operatorname{ap}(A) \subset S$.

ДокАЗАтЕльство. Первое включение очевидно. Чтобы доказать второе включение, предположим, что $\varphi \in \operatorname{ap}(A)$. Поскольку $\{\varphi \cdot \gamma \mid \gamma \in \Gamma\}$ предкомпактно в $A^{*}$, оно имеет конечную $\varepsilon$-сеть $\left\{\varphi \cdot \gamma_{1}, \ldots, \varphi \cdot \gamma_{n}\right\}$. С другой стороны, поскольку

$$
\left\|\widetilde{\varphi}_{s}-\widetilde{\varphi}_{t}\right\|_{\infty} \leqslant C_{\Gamma}\|\varphi \cdot s-\varphi \cdot t\|, \quad s, t \in \Gamma,
$$

отсюда следует, что $\left\{\widetilde{\varphi}_{\gamma_{1}}, \ldots, \widetilde{\varphi}_{\gamma_{n}}\right\}$ является $\varepsilon$-сетью для $\left\{\widetilde{\varphi}_{\gamma} \mid \gamma \in \Gamma\right\}$ и потому $\widetilde{\varphi} \in$ $\operatorname{AP}(\Gamma)$, т.е. $\varphi \in S$.

Пусть $K$-компакт, $M(K)$ - пространство комплексных регулярных борелевских мер в $K, M_{c}(K)$ - непрерывные меры из $M(K)$. Напомним [8, с. 52], что $K$ является вполне несовершенным тогда и только тогда, когда $M_{c}(K)=\{0\}$.

Заметим, что $M\left(\Sigma_{A}\right)$ можно рассматривать как подмножество $A^{*}$ относительно двойственности

$$
\langle\mu, a\rangle=\int_{\Sigma_{A}} \hat{a}(\varphi) d \mu(\varphi),
$$

где $a \in A$ и $\mu \in M\left(\Sigma_{A}\right)$.

ЛЕмма 2. Имеем $S \cap M_{c}\left(\Sigma_{A}\right)=\{0\}$.

ДокАЗАтЕльство. Предположим, что существует ненулевая $\mu \in S \cap M_{c}\left(\Sigma_{A}\right)$. Меру $\mu$ можно считать элементом $M(\widehat{\Gamma})$ с условием supp $\mu \subset \Sigma_{A}$. Тогда мы имеем

$$
\tilde{\mu}(\gamma)=\langle\mu, \gamma\rangle=\int_{\Sigma_{A}} \hat{\gamma}(\chi) d \mu(\chi)=\int_{\widehat{\Gamma}} \chi(\gamma) d \mu(\chi)=\mu^{\wedge}\left(\gamma^{-1}\right)
$$

где $\mu \hat{\mu}(\gamma)$ есть преобразование Фурье-Стилтьеса меры $\mu$. Пусть $\Phi$ есть инвариантное среднее в $\mathrm{AP}(\Gamma)$. Поскольку

$$
\langle\Phi, \chi\rangle= \begin{cases}1, & \chi=0 \\ 0, & \chi \neq 0\end{cases}
$$


где $\chi \in \widehat{\Gamma}$, то нетрудно видеть, что

$$
\left\langle\Phi, \overline{\chi(\gamma)} \mu^{\wedge}\left(\gamma^{-1}\right)\right\rangle=\mu\{\chi\}=0
$$

Это означает, что все коэффициенты Фурье-Бора функции $\mu^{\wedge}\left(\gamma^{-1}\right)$ равны нулю. По теореме единственности для почти периодических функций имеем $\mu^{\wedge}\left(\gamma^{-1}\right) \equiv 0$ и, следовательно, $\mu=0$.

Напомним, что $\omega^{*}$-спектр функционала $\varphi \in A^{*}$ определяется равенством $\sigma(\varphi)=$ $\overline{\varphi \cdot A}^{\omega^{*}} \cap \Sigma_{A}$. Нетрудно видеть, что $\sigma(\varphi)=h\left(I_{\varphi}\right)$, где $I_{\varphi}=\{a \in A \mid \varphi \cdot a=0\}-$ замкнутый идеал в $A$. Если обозначать через $G_{\chi}, \chi \in \widehat{\Gamma}$, коэффициенты Фурье-Бора функции $f \in \mathrm{AP}(\Gamma)$, то боровский спектр $\sigma_{B}(f)$ функции $f$ определяется как множество тех $\chi \in \widehat{\Gamma}$, для которых $G_{\chi}(f) \neq 0$. Хорошо известно, что если $f \in \operatorname{AP}(\Gamma)$, то $\sigma_{B}(f) \subset \sigma(f)$ и, более, того $\overline{\sigma_{B}(f)} \omega^{*}=\sigma(f)$.

Известная теорема Люмиса [9] утверждает, что функции из $L^{\infty}(\Gamma)$ с вполне несовершенным $\omega^{*}$-спектром являются почти периодическими.

ЛЕмма 3. Если $\Sigma_{A}$ вполне несовериенно, то $S=A^{*}$.

ДокАЗАТЕЛЬСТВо. По теореме Люмиса достаточно показать, что

$$
\sigma(\widetilde{\varphi}) \subseteq \operatorname{sp}(\omega) \quad \text { для всех } \varphi \in A^{*} .
$$

Пусть для некоторого $\varphi \in A^{*}$ найдется такое $\chi_{0} \in \sigma(\widetilde{\varphi})$, что $\chi_{0} \notin \operatorname{sp}(\omega)$. Тогда существует $f \in L^{1}(\Gamma)$ такое, что $\hat{f}\left(\chi_{0}\right) \neq 0$ и $\hat{f} \equiv 0$ в некоторой окрестности $\operatorname{sp}(\omega)$. Отсюда следует, что $\hat{f}_{s} \equiv 0(s \in \Gamma)$ в некоторой окрестности $\operatorname{sp}(\omega)$. Таким образом, $f_{s}(s \in \Gamma)$ принадлежит наименьшему идеалу в $L^{1}(\Gamma)$ с оболочкой $\operatorname{sp}(\omega)$ и потому $\omega\left(f_{s}\right)=0$. Нетрудно видеть, что $\widetilde{\varphi} * f=\left\langle\varphi, \omega\left(f_{s}\right)\right\rangle=0$ и потому $f \in I_{\widetilde{\varphi}}$. Поскольку $\chi_{0} \in \sigma(\widetilde{\varphi})$, имеем $\hat{f}\left(\chi_{0}\right)=0$, что противоречит $\hat{f}\left(\chi_{0}\right) \neq 0$.

Следующая лемма доказана в [4].

Лемма 4. Пусть $A-$ произвольная банахова алгебра. Если $A^{*}$ обладает свойством Шура, то ар $(A)=A^{*}$.

Ниже мы докажем теорему, которая по нашему мнению представляет самостоятельный интерес.

Теорема 2. Пусть $A$ - коммутативная банахова алгебра с ограниченной группой образующих. Если А регулярна по Аренсу, то

$$
S=\operatorname{ap}(A)=\overline{\operatorname{span}} \Sigma_{A}
$$

ДокАЗАТЕЛЬСТВо. Пусть Г - ограниченная группа (с дискретной топологией) образуюших в $A$. В силу леммы 1 достаточно показать, что

$$
S \subset \overline{\operatorname{span}} \Sigma_{A}
$$

Пусть $F \in A^{* *}$ такое, что $\langle F, \chi\rangle=0$ для всех $\chi \in \Sigma_{A}$. Нам надо доказать, что $\langle F, \varphi\rangle=0$ для всех $\varphi \in S$. Пусть $G$ - боровская компактификация групшы $\Gamma, \bar{f}(g)(g \in G)-$ 
боровскоепродолжение $f \in \mathrm{AP}(\Gamma)$. Боровское продолжение характеров $\chi(\gamma)$ из $\widehat{\Gamma}$ будем обозначать через $\chi(g)(g \in G)$.

Для $\varphi \in S$ и $g \in G$ определим $\varphi \cdot g \in A^{*}$ следующим образом: в силу плотности $\Gamma$ в $G$ имеется направленность $\left(\gamma_{\lambda}\right)$ в $\Gamma$ такая, что $\gamma_{\lambda} \rightarrow g$ в $G$. Заметим, что $\left(\varphi \cdot \gamma_{\lambda}\right)$ равномерно ограничено и, кроме того,

$$
\lim _{\lambda}\left\langle\varphi \cdot \gamma_{\lambda}, a\right\rangle=\overline{\widetilde{\varphi \cdot a}}(g), \quad a \in A .
$$

Это дает возможность определить $\varphi \cdot g \in A^{*}$ как

$$
\varphi \cdot g=\omega^{*}-\lim _{\lambda} \varphi \cdot \gamma_{\lambda}
$$

По определению имеем

$$
\langle\varphi \cdot g, a\rangle=\overline{\widetilde{\varphi \cdot a}}(g) .
$$

Из равенства (1) ясно, что $\varphi \cdot g$ является единственной слабо предельной точкой множества $\left(\varphi \cdot \gamma_{\lambda}\right)$. С другой стороны, поскольку $A$ регулярно по Аренсу, $\left(\varphi \cdot \gamma_{\lambda}\right)$ слабо предкомпактно в $A^{*}$ и потому

$$
\varphi \cdot g=\omega-\lim _{\lambda} \varphi \cdot \gamma_{\lambda}
$$

Значит, $\{\varphi \cdot g \mid g \in G\}$ слабо предкомпактно в $A^{*}$ (в силу компактности $G$ оно предкомпактно и по норме). Теперь, используя (2) подобным же образом, получим, что $g \rightarrow \varphi \cdot g$ слабо непрерьвно в $G$. Отсюда следует, что $\overparen{F \cdot \varphi} \in \operatorname{AP}(\Gamma)$ и, следовательно, $F \cdot \varphi \in S$. Заметим также, что

$$
\langle F, \varphi \cdot g\rangle=\widetilde{\widetilde{F \cdot \varphi}}(g), \quad g \in G .
$$

А теперь найдем коэффициенты Фурье-Бора функции $\widetilde{F \cdot \varphi}$. В силу слабой непрерьвности отображения $g \rightarrow \varphi \cdot g$ в $G$ существует векторнозначный интеграл

$$
\varphi_{\chi}=\int_{G} \overline{\chi(g)}(\varphi \cdot g) d g .
$$

Далее, нетрудно видеть, что для произвольного $a \in A$

$$
C_{\chi}(\widetilde{\varphi \cdot a})=\hat{a}(\chi) C_{\chi}(\widetilde{\varphi})
$$

и потому имеем

$$
\begin{aligned}
\left\langle\varphi_{\chi}, a\right\rangle & =\int_{G} \overline{\chi(g)}\langle\varphi \cdot g, a\rangle d g=\int_{G} \overline{\chi(g)} \overline{\overline{\varphi \cdot a}}(g) d g \\
& =C_{\chi}(\widetilde{\varphi \cdot a})=\hat{a}(\chi) C_{\chi}(\tilde{\varphi}) .
\end{aligned}
$$

Следовательно, $\varphi_{\chi}=\chi C_{\chi}(\tilde{\varphi})$. Используя $(3)$, получим, что

$$
\begin{aligned}
C_{\chi}(\widetilde{F \cdot \varphi}) & =\int_{G} \overline{\chi(g)} \overline{\widetilde{F \cdot \varphi}}(g) d g=\int_{G} \overline{\chi(g)}\langle F, \varphi \cdot g\rangle d g \\
& =\left\langle F, \varphi_{\chi}\right\rangle=\langle F, \chi\rangle C_{\chi}(\tilde{\varphi}) .
\end{aligned}
$$

Таким образом, мы имеем

$$
C_{\chi}(\widetilde{F \cdot \varphi})=\langle F, \chi\rangle C_{\chi}(\tilde{\varphi})
$$

Поскольку $\sigma_{B}(\widetilde{F \cdot \varphi}) \subset \Sigma_{A}$ и $\langle F, \chi\rangle=0$ для всех $\chi \in \Sigma_{A}$, из последнего равенства получим, что все коэффициенты Фурье-Бора функций $\widetilde{F \cdot \varphi}$ равны нулю. По теореме единственности имеем $\overline{F \cdot \varphi} \equiv 0$ или $\langle F, \varphi \cdot \gamma\rangle=0$ для всех $\gamma \in \Gamma$. В частности, получим, что $\langle F, \varphi\rangle=0$ для всех $\varphi \in S$. Теорема доказана.

Отметим одно следствие этой теоремы. 
СледствИЕ 1. Пусть $A$ - регулярная по Аренсу коммутативная банахова алгебра с ограниченной группой образующих. Если $\Sigma_{A}$ вполне несовершенно, то

$$
A^{*}=\overline{\operatorname{span}} \Sigma_{A} .
$$

ДокАЗАТЕЛЬСТво. В силу леммы 3 имеем $S=A^{*}$ и в силу теоремы $2 S=\overline{\operatorname{span}} \Sigma_{A}$.

ТЕОрема 3. Если $A$ - коммутативная банахова алгебра с ограниченной группой образующ, $и$, то

$$
\operatorname{ap}(A)=\overline{\operatorname{span}} \Sigma_{A}
$$

ДокАЗАТЕЛьСтво. Доказательство проводится аналогично доказательству теоремы 2 с заменой $S$ на $\operatorname{ap}(A)$. Надо учесть, что при такой замене мы не нуждаемся в условии регулярности по Аренсу.

А теперь докажем основную теорему.

ДоКАЗАТЕЛЬСТво ТЕОРЕМЫ 1. Предположим что $A^{*}$ обладает свойством Шура.

а) В силу леммы 4 имеем $\operatorname{ap}(A)=A^{*}$. По лемме $1 S=A^{*}$. Применяя лемму 2 , получим, что $M_{c}\left(\Sigma_{A}\right)=\{0\}$. Это означает, что $\Sigma_{A}$ - вполне несовершенное множество [8, c. 52].

b) Поскольку $\operatorname{ap}(A)=A^{*}$, в силу теоремы 3 получим, что $A^{*}=\overline{\operatorname{span}} \Sigma_{A}$.

c) Чтобы доказать, что $A^{*}$ обладает свойством Радона-Никодима, достаточно показать, что любое сепарабельноеподпространство пространства $A^{*}$ содержится в некотором сепарабельном сопряженном пространстве [1, с. 81-82]. Пусть $M$ - сепарабельное подпространство пространства $A^{*}$ и $\left(\varphi_{1}, \varphi_{2}, \ldots\right)$ - плотное счетное подмножество в $M$. В силу b) $A^{*}=\overline{\operatorname{span}} \Sigma_{A}$. Поэтому $\varphi_{i}=\lim _{j} \varphi_{i j}$, где $\varphi_{i j}(i, j=1,2, \ldots)$ есть конечная линейная комбинация характеров из $\Sigma_{A}$.

Положим

$$
I_{i j}=\left\{a \in A \mid \varphi_{i j} \cdot a=0\right\} \quad \text { и } \quad I=\bigcap_{i, j=1}^{\infty} I_{i j}
$$

Заметим, что $M \subset I^{\perp}$. Действительно, если $a \in I$ и $\varphi \in M$, то $\varphi_{i j} \cdot a=0$, что влечет $\left\langle\varphi_{i}, a\right\rangle=0(i=1,2, \ldots)$ и потому $\langle\varphi, a\rangle=0$.

Очевидно, что

$$
h(I)={\overline{\bigcup_{i, j=1}^{\infty} \sigma\left(\varphi_{i j}\right)}}^{\omega^{*}}
$$

Поскольку $\sigma\left(\varphi_{i j}\right)(i, j=1,2, \ldots)$ конечно, из последнего равенства получим, что $h(I)$ есть сепарабельное подмножество в $\Sigma_{A}$. В силу а) $\Sigma_{A}$ есть вполне несовершенное множество и потому $h(I)$ счетно. А теперь заметим следующее:

1) $A / I$ имеет ограниченную группу образующих;

2) $A / I$ регулярна по Аренсу;

3) $\Sigma_{A / I}=h(I)$ счетно. 
По следствию 1 имеем

$$
(A / I)^{*}=\overline{\operatorname{span}} h(I) .
$$

Следовательно, $(A / I)^{*}$ сепарабельно и $M \subset I^{\perp}=(A / I)^{*}$.

Теорема доказана.

Отметим, одно следствие этой теоремы.

Пусть $A$-произвольная комплексная банахова алгебра с единищей $e$. Элемент $a \in A$ называется әрмитовым, если $\|\exp (i t a)\|=1$ для всех $t \in \mathbb{R}$. Обозначим через $A_{a}$ замкнутую подалгебру $A$, порожденную $a$ и $e$. Нетрудно видеть, что

$$
A_{a}=\overline{\operatorname{span}}(\exp (i t a) \mid t \in \mathbb{R}),
$$

т.е. $A_{a}$ имеет ограниченную группу образуюших. Также заметим, что пространство Гельфанда алгебры $A_{a}$ отождествляется со спектром $\sigma(a)$ элемента $a$.

СледСтвиЕ 2. Если $A_{a}^{*}$ обладает свойством Шура, то

a) $\sigma($ а) - счетное множество;

b) $A_{a}^{*}=\overline{\operatorname{span}}\left(\varphi_{\lambda} \mid \lambda \in \sigma(a)\right)$, əде $\left\langle\varphi_{\lambda}, \exp (i t a)\right\rangle=\exp (i t \lambda)$;

c) $A_{a}^{*}$ обладает свойством Радона-Никодима.

\section{СПИСОК ЦИТИРОВАННОЙ ЛИТЕРАТУРЫ}

[1] Diestel J., Uhl J. J. Vector Measures. Math. Surveys. V. 15. Providence, R.I.: Amer. Math. Soc., 1977.

[2] Lust-Piquard F. L'Espace des fonctions presque-periodiques dont le spectre est contenu dans un ensemble compact denombrable a la propriete de Schur // Colloq. Math. 1979. V. 41. № 2 . P. 273-284.

[3] Lust-Piquard F. Means on $C V_{p}(G)$, subspace of $C V_{p}(G)$ with RNP and Schur property // Ann. Inst. Fourier (Grenoble). 1989. V. 39. № 4. P. 969-1006.

[4] Mustafayev H., Ülger A. A class of Banach algebras whose duals have the Schur property // Tr. J. Math. 1999. V. 23. № 3. P. 441-452.

[5] Arens R. The adjoint of a bilinear operation // Proc. Amer. Math. Soc. 1951. V. 2. P. 839-848.

[6] Duncan J., Hosseiniun S. A. R. The second dual of a Banach algebra // Proc. Royal Soc. Edinburgh. Ser. A. 1979. V. 84. P. 309-325.

[7] Брудный Ю. А., Горин Е. А. Изометрические представления и дифференциальные неравенства. Ярославль: ЯГУ, 1981.

[8] Lacey H. The Isometric Theory of Classical Banach Spaces. New York-Heidelberg: SpringerVerlag, 1974.

[9] Loomis L.H. The spectral characterization of a class of almost periodic functions // Ann. Math. 1960. V. 72. P. 362-368.

Институт математики и механики АН Азербайджана, г. Баку

Поступило 08.09.1999

Исправленный вариант 10.10 .2001 